\title{
THE NONIMBEDDABILITY OF REAL HYPERSURFACES IN SPHERES
}

\author{
JAMES J. FARAN V
}

(Communicated by Irwin Kra)

\begin{abstract}
It is shown that there exist real analytic real hypersurfaces in $\mathbf{C}^{n}$ which cannot be locally holomorphically imbedded in any finite dimensional sphere $S^{2 N-1} \subset \mathbf{C}^{2 N}$.
\end{abstract}

In [1], Chern and Moser developed a theory of local invariants of real hypersurfaces in complex manifolds. In doing so, they stressed the analogies between pseudoconformal geometry and Riemannian geometry, defining analogues of the Riemannian curvature tensor, the Levi-Civita connection, geodesics, etc. These analogies are in general quite weak (e.g., "geodesics" may spiral, see [2]). The purpose of this note is to show that there is another case where the analogy breaks down.

It is a classical result that any analytic metric can be locally induced by the flat metric, i.e., given any analytic metric there exists locally an isometric imbedding into some Euclidean space. The corresponding theorem in pseudoconformal geometry would state that every analytic real hypersurface could be (locally) holomorphically imbedded in the unit sphere in some $\mathbf{C}^{n}$. The two theorems of this paper give counterexamples to this.

It should be noted that if a global holomorphic imbedding of the boundary of a strictly pseudoconvex domain into a unit sphere exists, it naturally extends to a proper holomorphic mapping of the whole domain into the unit ball. Lempert [4] has shown that any smoothly bounded strictly pseudoconvex domain can be properly and holomorphically imbedded in the unit ball in infinite dimensional space (i.e., $l_{2}$ ). The results here indicate that Lempert's theorem cannot be extended to imbeddings in finite dimensional space.

THEOREM. Given integers $N \geq n \geq 2$, there exists a smooth, strictly pseudoconvex, real analytic (in fact, polynomial) real hypersurface $M$ in $\mathbf{C}^{n}$ that cannot be locally analytically imbedded in the unit sphere in $\mathbf{C}^{N}$.

PROOF. We shall examine the problem of mapping a real hypersurface $M$ into $\mathrm{C}^{N}$ so that the image is tangent to the sphere to order $d$. It will suffice to show that by taking $d$ large enough, we can find a real hypersurface $M$ that cannot be imbedded in $\mathbf{C}^{N}$, tangent to the unit sphere to order $d$.

Let $V$ be the space of real-valued polynomials $F\left(z^{\prime}, \bar{z}^{\prime}, u\right)$ of degree less than or equal to $d$ that vanish at $(0,0,0), W$ the space of complex polynomials $f^{\alpha}\left(z^{\prime}, w\right)$, $\alpha=1, \ldots, N$, of degree less than or equal to $d$ such that $\sum_{\alpha}\left|f^{\alpha}(0,0)\right|^{2}-1=0$.

Received by the editors March 25, 1987 and, in revised form, July 6, 1987.

1980 Mathematics Subject Classification (1985 Revision). Primary 32F25; Secondary 53B25, $53 \mathrm{~B} 15$.

(C) 1988 American Mathematical Society $0002-9939 / 88 \$ 1.00+\$ .25$ per page 
(We have let $z^{\prime}=\left(z_{1}, \ldots, z_{n-1}\right), w=u+i v=z_{n}$.) $V$ corresponds to the set of $d$-jets of real hypersurfaces $v=F(z, \bar{z}, u)$ containing the origin and $W$ corresponds to the set of $d$-jets of imbeddings $\mathbf{C}^{n} \rightarrow \mathbf{C}^{N}$ sending the origin into the unit sphere. $\operatorname{dim} V=\left(\begin{array}{c}2 n-1+d \\ d\end{array}\right), \operatorname{dim}_{\mathbf{R}} W=2 N\left(\begin{array}{c}n+d \\ d\end{array}\right)-1$. Define $\Phi: V \times W \rightarrow V$ by

$$
\Phi\left(F, f^{\alpha}\right)=d \text {-jet of } \sum_{\alpha} \mid f^{\alpha}\left(z^{\prime}, u+\left.i F(z, \bar{z}, u)\right|^{2}-1 .\right.
$$

If every real hypersurface $M \subset \mathbf{C}^{n}$ is imbeddable in the unit sphere in $\mathbf{C}^{N}$ to order $d$, then for every $F \in V$, there exists $f^{\alpha} \in W$ such that $\Phi\left(F, f^{\alpha}\right)=0$.

LEMMA. There exists $\left(F_{0}, f_{0}^{\alpha}\right) \in \Phi^{-1}(0) \subset V \times W$ such that the derivative $D \Phi\left(F_{0}, f_{0}^{\alpha}\right): V \times W \rightarrow V$ is surjective.

Postponing the proof of this, notice that this implies that in a neighborhood of $\left(F_{0}, f_{0}^{\alpha}\right), \Phi^{-1}(0)$ is a smooth manifold of $V \times W$ of $\operatorname{dimension} \operatorname{dim} W$. If we have imbeddability up to order $d$ though, we have seen that the dimension of $\Phi^{-1}(0)$ must be at least $\operatorname{dim} V$. So imbeddability up to order $d$ implies

$$
\operatorname{dim} W=2 N \frac{(n+d) !}{n ! d !} \geq \frac{(2 n-1+d) !}{(2 n-1) ! d !}=\operatorname{dim} V
$$

The left-hand side is a polynomial in $d$ of degree $n$, the right-hand side a polynomial of degree $2 n-1$, so this inequality cannot hold for arbitrarily large orders $d$. Thus we cannot have imbeddability to arbitrarily high order. (Note that in the case $n=N=2$, the inequality becomes $d \leq 9$. This was obtained by Poincaré[3].)

To complete the proof of the theorem, it suffices to provide a

PROOF OF THE LEMMA. Let $f_{0}^{\alpha}$ be an imbedding of the real hypersurface $v=F_{0}$ in the unit sphere. By making a linear change of coordinates in $\mathbf{C}^{n}$ and a unitary change of coordinates in $\mathbf{C}^{N}$, we may assume $f^{n}(0,0)=1$ and $d F(0,0,0)=0$. Since $\left(f^{1}, \ldots, f^{n}\right)$ is an imbedding, the vector $\partial / \partial w$ (which is tangent to $M$ but not in the maximal complex tangent space) must be mapped by $f_{*}^{\alpha}(0,0)$ to a vector tangent to $S^{2 N-1}$ but not in its maximal complex tangent space. It follows that $i \partial f^{n} / \partial w(0,0) \neq 0$ is real.

The directional derivative of $\Phi$ at $\left(F_{0}, f_{0}^{\alpha}\right)$ in the direction $(G, 0) \in V \times W$ is

$$
D \Phi\left(F_{0}, f_{0}^{\alpha}\right)(G, 0)=d \text {-jet of }\left.G \operatorname{Re}\left[i \sum_{\alpha} \frac{\partial f^{\alpha}}{\partial w} \overline{f^{\alpha}}\right]\right|_{w=u+i F} .
$$

By the above, Re $\left.i\left[\sum_{\alpha}\left(\partial f^{\alpha} / \partial w\right) \overline{f^{\alpha}}\right]\right|_{w=u+i F}$ is nonvanishing at the origin. Therefore $D \Phi\left(F_{0}, f_{0}^{\alpha}\right): V \times\{0\} \rightarrow V$ is surjective.

This shows that we cannot always obtain an imbedding if we choose $N$ beforehand. But more is true.

THEOREM. Given an integer $n$, there exists a smooth, strictly pseudoconvex, analytic real hypersurface $M$ defined in a neighborhood of the origin such that for every integer $N \geq n$ there does not exist a holomorphic imbedding $f: \mathbf{C}^{n} \rightarrow \mathbf{C}^{N}$ defined in some neighborhood of the origin taking $M$ into the unit sphere in $\mathbf{C}^{N}$.

ProOF. Start with a polynomial $F_{1}\left(z^{\prime}, \bar{z}^{\prime}, u\right)$ of degree $d_{1}$, so that $v=F_{1}\left(z^{\prime}, \bar{z}^{\prime}, u\right)$ defines a strictly pseudoconvex real hypersurface $M_{1}$ in a neighborhood of the origin (and $F_{1}(0,0,0)=0$ ). If $M_{1}$ cannot be imbedded in any $S^{2 N-1}$, we are done. If 
$M$ can be imbedded in some $S^{2 N-1}$, pick an $N_{2}$ and a holomorphic imbedding $\Phi_{1}: M_{1} \rightarrow S^{2 N_{2}-1}$. By the arguments given above, we can choose $d_{2}>d_{1}$ so that there exist polynomials of degree $d_{2}$ arbitrarily close to $F_{1}\left(z^{\prime}, \bar{z}^{\prime}, u\right)$, agreeing with $F_{1}$ up through degree $d_{1}$, which define real hypersurfaces which cannot be holomorphically imbedded in $S^{2 N_{2}-1}$ up to order $d_{2}$ (choose $d_{2}$ so that

$$
2 N_{2} \frac{\left(n+d_{2}\right) !}{d_{2} !}<\frac{\left(2 n-1+d_{2}\right) !}{(2 n-1) ! d_{2} !}-\frac{\left(2 n-1+d_{1}\right) !}{(2 n-1) ! d_{1} !} \text {. }
$$

Thus we obtain a polynomial $F_{2}\left(z^{\prime}, \bar{z}^{\prime}, u\right)$. Proceeding, we obtain an $N_{3}$ so that $\left\{v=F_{2}\right\}$ imbeds in $S^{2 N_{3}-1}$; a polynomial $F_{3}$ of degree $d_{3}$ which defines a real hypersurface which cannot be imbedded to order $d_{3}$ in $S^{2 N_{3}-1}$, and so on. If at any stage we cannot find an $N_{k}$ we are done. We can always find $d_{k}$ and $F_{k}$ by the arguments used to prove the previous theorem. If we let $a_{j_{1}, \ldots, j_{n-1}, l_{1}, \ldots, l_{n-1} m}^{k}$ be the coefficient of $z_{1}^{j_{1}} \cdots z_{n-1}^{j_{n-1}} \bar{z}_{1}^{l_{1}} \cdots \bar{z}_{n-1}^{l_{n-1}} u^{m}$ in $F_{k+1}\left(z^{\prime}, \bar{z}^{\prime}, u\right)-F_{k}\left(z^{\prime}, \bar{z}^{\prime}, u\right)$, it is clear that we can choose $F_{k+1}$ so that

$$
\left|a_{j_{1}, \ldots, j_{n-1}, l_{1}, \ldots, l_{n-1} m}^{k}\right| \leq \frac{1}{j_{1} ! \cdots j_{n-1} ! l_{1} ! \cdots l_{n-1} ! m !} .
$$

It follows that $\lim _{k \rightarrow \infty} F_{k}$ exists and is a convergent power series $F\left(z^{\prime}, \bar{z}^{\prime}, u\right)$, and that $v=F\left(z^{\prime}, \bar{z}^{\prime}, u\right)$ defines a strictly pseudoconvex real hypersurface in a neighborhood of the origin. Suppose $\{v=F\}$ can be imbedded in $S^{2 N-1}$ by a map $\phi$. Since $N_{k}$ is a strictly increasing sequence we can choose $k$ so large that $N_{k} \geq N$. Then $\phi$ imbeds $v=F_{k}\left(z^{\prime}, \bar{z}^{\prime}, u\right)$ in $S^{2 N-1}$ to order $d_{k}$. By imbedding $S^{2 N-1}$ in $S^{2 N_{k}-1}$ in the usual way, we get an imbedding of $\left\{v=F_{k}\right\}$ into $S^{2 N_{k}-1}$ to or$\operatorname{der} d_{k}$. However, we constructed $F_{k}$ precisely so that this was not possible. This contradiction completes the proof of the theorem.

\section{REFERENCES}

1. S.-S. Chern and J. K. Moser, Real hypersurfaces in complex manifolds, Acta Math. 133 (1974), 219-271.

2. C. Fefferman, Monge-Ampère equations, the Bergman kernel, and the geometry of strictly pseudoconvex domains, Ann. of Math. (2) 103 (1976), 395-416.

3. H. Poincaré, Les fonctions analytiques de deux variables et la represéntation conforme, Rend. Circ. Mat. Palermo 23 (1907), 185-220.

4. L. Lempert, Imbedding strictly pseudoconvex domains into a ball, Amer. J. Math. 104 (1982), 901-904.

Department of Mathematics, State University of New York at Buffalo, BUfFALO, NEW YORK 14214 